\title{
Human mesenchymal stem cells target adhesion molecules and receptors involved in T cell extravasation
}

Federica Benvenuto ${ }^{1,2}$, Adriana Voci $^{3}$, Enrico Carminati ${ }^{3}$, Francesca Gualandi ${ }^{4}$, Gianluigi Mancardi ${ }^{1,2}$, Antonio Uccelli $i^{1,2 \dagger}$ and Laura Vergani ${ }^{3 *+}$

\begin{abstract}
Introduction: Systemic delivery of bone marrow-derived mesenchymal stem cells (MSC) seems to be of benefit in the treatment of multiple sclerosis (MS), an autoimmune disease of the central nervous system (CNS) sustained by migration of $T$ cells across the brain blood barrier (BBB) and subsequent induction of inflammatory lesions into CNS. MSC have been found to modulate several effector functions of T cells. In this study, we investigated the effects of MSC on adhesion molecules and receptors on T cell surface that sustain their transendothelial migration.

Methods: We used different co-culture methods combined with real-time PCR and flow cytometry to evaluate the expression both at the mRNA and at the plasma-membrane level of a4 integrin, $\beta 2$ integrin, ICAM- 1 and CXCR3. In parallel, we assessed if MSC are able to modulate expression of adhesion molecules on the endothelial cells that interact with $T$ cells during their transendothelial migration.

Results: Our in vitro analyses revealed that MSC: (i) inhibit proliferation and activation of both peripheral blood mononuclear cells (PBMC) and $\mathrm{CD}^{+}$-selected lymphocytes through the release of soluble factors; (ii) exert suppressive effects on those surface molecules highly expressed by activated lymphocytes and involved in transendothelial migration; (iii) inhibit CXCL10-driven chemotaxis of $\mathrm{CD}^{+}$cells; (iv) down-regulated expression of adhesion molecules on endothelial cells.

Conclusions: Taken together, these data demonstrate that the immunosuppressive effect of MSC does not exclusively depends on their anti-proliferative activity on T cells, but also on the impairment of leukocyte migratory potential through the inhibition of the adhesion molecules and receptors that are responsible for $T$ cell trafficking across BBB. This could suggest a new mechanism through which MSC modulate T cell responses.
\end{abstract}

Keywords: mesenchymal stem cells, $\mathrm{CD}^{+}$-selected lymphocytes, human endothelial cells, immunosuppressive effects, surface adhesion molecules and receptors, transendothelial migration, leukocyte migratory potential

\section{Introduction}

The central nervous system (CNS) is an immune-privileged site where the endothelial blood-brain barrier (BBB) tightly controls lymphocyte entry. Under physiological conditions, lymphocyte traffic across the BBB is low, but during inflammatory diseases of the CNS-such as multiple sclerosis (MS) or its animal model, experimental autoimmune

\footnotetext{
* Correspondence: Laura.Vergani@unige.it

${ }^{\dagger}$ Equal contributors

${ }^{3}$ Department of Earth, Environment and Life Sciences (DISTAV), University of Genoa, Corso Europa 26, 16132 Genova, Italy

Full list of author information is available at the end of the article
}

encephalomyelitis (EAE) - circulating $\mathrm{CD}^{+}$and $\mathrm{CD} 8^{+}$lymphocytes gain access to the CNS leading to inflammation, demyelination, and neurodegeneration [1-3]. Preventing the migration of lymphocytes into the CNS seems to be an important therapeutic approach for MS, and adhesion molecules on lymphocytes have long been studied as possible targets $[4,5]$ possibly leading to the translation into relevant therapies for MS [6].

Integrins are one of the major families of adhesion molecules that mediate binding of leukocytes to vascular endothelium leading to their extravasation [7], and they are probably involved in demyelinating diseases [8-10]. 
Integrins typically consist of $\alpha$ and $\beta$ subunits [7]; $\alpha 4$ integrin interacts with $\beta 1$ integrin forming the very late antigen-4 (VLA-4) antigen expressed on $\mathrm{T}$ and $\mathrm{B}$ cells. VLA-4 is responsible for lymphocyte binding to endothelial cells $[8,9]$; the ability of $\mathrm{T}$ cells to induce EAE seems to correlate with $\alpha 4$ integrin expression $[10,11]$. In addition, the monoclonal antibody (mAb) natalizumab (NTZ), developed to treat relapsing-remitting MS, selectively binds $\alpha 4$ integrin on autoreactive T cells and thus reduces their extravasation into CNS [12]. Also $\beta 2$ integrin acts in leukocyte trafficking as a constituent of the lymphocyte function-associated antigen-1 (LFA-1) that binds the intercellular adhesion molecules (ICAMs) on endothelial cells [3]. Other molecules seem to be involved in the pathogenesis of MS. In particular, ICAM-1, a member of the Immunoglobulin Superfamily (IgSF) that is weakly expressed on resting lymphocytes but highly expressed on endothelial cells [13, 14], is induced by inflammatory mediators [14-16] and acts in transmigration of $\mathrm{T}$ cells, of T-helper type (Th) 17 lymphocytes in particular [17]. Also the chemokine receptor CXCR3 on the lymphocyte surface seems to act in the patrolling of the CNS [18] and may play a role in MS pathogenesis [19] because it triggers rapid leukocyte adhesion and is induced in activated $\mathrm{CD} 4^{+}$and $\mathrm{CD} 8^{+} \mathrm{T}$ cells crossing the BBB [18-20].

The molecular counterparts for lymphocyte trafficking into the CNS are the adhesion molecules on endothelial cells of the BBB, whose expression typically increases under inflammatory conditions [21]. Among these, the most important are ICAM-1 and the activated leucocyte cell adhesion molecule (ALCAM) that are upregulated by inflammatory stimuli $[22,23]$. In particular, postmortem sections from patients with MS and mice with EAE showed higher expression of ALCAM. Moreover, mAbs against ALCAM reduced the accumulation of $\mathrm{CD} 4^{+} \mathrm{T}$ cells, decreased the disease severity, and delayed the onset of EAE [22, 23]. For this reason, ALCAM has been recently associated with risk, development, and progression of MS [24].

Mesenchymal stem cells (MSC) are progenitor cells of mesodermal origin that can be found in almost every connective tissue, including the bone marrow where they have been well characterized [25]. MSC are capable of modulating immune responses [26, 27] by having an anti-apoptotic effect on different cell types including $\mathrm{T}$ lymphocytes [28] and neurons $[29,30]$, as well as by releasing trophic factors and favoring endogenous neurogenesis [29-31]. MSC have therefore emerged as a promising tool for therapeutic applications in neurological experimental conditions, such as for the treatment of EAE, through inducing T-cell tolerance against myelin antigens, reducing T-cell infiltrates in the CNS [32], inhibiting the encephalitogenic potential of autoreactive $\mathrm{T}$ cells and axonal loss
[33], and protecting from oxidative stress damage [31, 34]. Since the 1990s, MSC have been used in clinical settings in humans to promote the engraftment of hematopoietic stem cells (HSC) $[35,36]$ and to treat immune-mediated diseases such as acute graft versus host disease (GvHD) [37]. Some studies demonstrated that MSC inhibit cell proliferation and decrease interferon gamma (IFN $\gamma$ ) production in T cells, probably through the secretion of soluble factors but also by "cell to cell contact" mechanisms [28, 38, 39]. In addition, soluble proinflammatory factors released by activated lymphocytes strongly influence MSC modulatory activity, supporting the idea of an intense cross-talk between MSC and immune cells [40, 41]. Despite the large amount of studies, little is known on the ability of MSC to affect the expression of molecules sustaining the lymphocyte-BBB interaction necessary for the lymphocytes to gain access to inflamed tissues.

Here we hypothesized that MSC may modulate the capability of activated lymphocytes to cross the BBB and reach the CNS, at least in part, by selectively acting on specific adhesion molecules and chemokine receptors involved in the extravasation of activated $\mathrm{T}$ cells. In the light of their possible implication in pathogenesis of MS, we explored the ability of human MSC to modulate the expression, both at the mRNA level and at the plasmamembrane level, of $\alpha 4$ integrin, $\beta 2$ integrin, CXCR3, and ICAM-1 on $\mathrm{CD}^{+}$lymphocytes, and of ICAM-1 and ALCAM on endothelial cells.

\section{Methods \\ Chemicals}

All chemicals, unless otherwise indicated, were of analytical grade and were obtained from Sigma-Aldrich Corp. (Milan, Italy).

\section{Human mesenchymal stem cell isolation and cell culture}

Human bone marrow samples were obtained from healthy donors undergoing bone marrow explant from allogeneic transplantation procedures. Informed consent, approved by the local Ethics Committee (Regione Liguria), was obtained from all donors. Donors' age ranged between 22 and 54 years $($ mean $=38.6)$. Mononuclear cells were isolated by density gradient centrifugation $(1.077 \mathrm{~g} / \mathrm{ml}$, Lympholyte Cell separation Media; Cedarlane Laboratories Ltd, Burlington, Ontario, Canada), and cultured in Human Mesencult Basal Medium additioned with its specific supplement (Stem Cell Technologies, Vancouver, BC, Canada) at $37{ }^{\circ} \mathrm{C}$ in a humidified $5 \% \mathrm{CO}_{2}$ atmosphere. MSC growing as adherent cells were harvested at $80 \%$ of confluence using Trypsin $0.05 \%$-ethylenediamine tetraacetic acid (EDTA) $0.02 \%$ and then expanded. MSC were characterized by flow cytometry at each passage and used upon phenotypic characterization as $\mathrm{CD} 73^{+}, \mathrm{CD} 44^{+}, \mathrm{CD} 105^{+}$, $\mathrm{CD}^{\circ} 0^{+}, \mathrm{CD} 45^{-}, \mathrm{CD} 34^{-}$, and $\mathrm{CD} 14^{-}$cells [28]. For each 
experiment we used the BM-derived MSC from a single donor, and the experiment was repeated using MSC from different donors.

\section{Peripheral blood mononuclear cell isolation, $\mathrm{CD}^{+}$ lymphocyte selection, and coculture with MSC}

Peripheral blood mononuclear cells (PBMC) were separated by density gradient centrifugation from heparinized blood of healthy donors after informed consent [28]. PBMC were resuspended in RPMI 1640 supplemented with L-glutamine, penicillin, streptomycin and $10 \%$ fetal calf serum (FCS) and dispensed in microtiter $200 \mu$ plates (Sarstedt S.r.l, Verona, Italy) at a concentration of $1 \times 10^{5}$ cells/well. Upon stimulation with $2 \mu \mathrm{g} /$ ml anti-Human OKT3 (R\&D Systems, Milan, Italy) and $1 \mu \mathrm{g} / \mathrm{ml}$ anti-Human CD28 (BD Biosciences, San Jose, CA, USA) antibodies, PBMC were cultured alone or in the presence of MSC from different donors at three different PBMC/MSC ratios (20:1, 10:1, and 4:1). After 4 days, cells were harvested and used for experiments.

$\mathrm{CD}^{+}$lymphocytes were isolated from the PBMC by magnetic negative selection using a Pan $\mathrm{T}$ isolation kit (Miltenyi Biotech GmBh, Bergish Gladbach, Germany). Briefly, PBMC were resuspendend in RPMI without FCS, plated at a concentration of $2 \times 10^{6}$ cells $/ \mathrm{ml}$, and maintained for 5 minutes at $37{ }^{\circ} \mathrm{C}$ to allow the adhesion of monocytes. The monocyte-depleted cells were then harvested, resuspended in phosphate-buffered saline (PBS)$0.02 \%$ EDTA at a concentration of $10^{7}$ cells $/ 40 \mu \mathrm{l}$, and incubated with $10 \mu \mathrm{l}$ Biotin Antibody Cocktail for $10 \mathrm{mi}$ nutes at $4{ }^{\circ} \mathrm{C}$. After incubation with anti-Biotin microbeads for 15 minutes, cells were washed, resuspended in PBS$0.02 \%$ EDTA $\left(10^{7}\right.$ cells $\left./ 500 \mu \mathrm{l}\right)$, and applied on MS columns (Miltenyi Biotech $\mathrm{GmBh}$ ) following the manufacturers' instructions. $\mathrm{CD}^{+}$cells were then employed in transwell experiments using flat-bottom microtiter plates (Millipore, Milan, Italy). $\mathrm{CD}^{+}$cells were dispensed at a concentration of $1 \times 10^{5}$ cells/well in the lower chamber previously coated with $\alpha \mathrm{CD} 3(10 \mu \mathrm{g} / \mathrm{ml})$ and $\alpha$ CD28 $(1 \mu \mathrm{g} /$ $\mathrm{ml}$ ) mAbs. MSC were seeded in the upper chamber and three different $\mathrm{CD}^{+} / \mathrm{MSC}$ ratios were used (20:1, 10:1, and 4:1). After 4 days, cells were harvested and used for experiments.

For all treatments we checked the viability of cells by Trypan Blue staining, which resulted to be over $95 \%$ also at the highest coculture ration with MSC ratio (4:1).

\section{Human endothelial cell culture and expansion}

Human endothelial cells (HECV; Cell Bank and Culture in GMP-IST, Genoa, Italy) were maintained at $37{ }^{\circ} \mathrm{C}$ in a humidified $5 \% \mathrm{CO}_{2}$ atmosphere in Dulbecco's modified Eagle's medium high glucose (DMEM) supplemented with L-glutamine, penicillin, streptomycin and $10 \%$ FCS. HECV were characterized by flow cytometry as $\mathrm{CD} 166^{+}, \mathrm{CD} 105^{+}$,
$\mathrm{CD} 54^{+}, \mathrm{CD} 146^{+} \mathrm{MHC}$ class I, CD102-, and CD106 ${ }^{-}$cells. In coculture experiments, HECV were dispensed at a concentration of $1 \times 10^{6}$ cells/well using flat-bottom 24-well plates and stimulated with recombinant human IFN $\gamma(200$ IU/ml; Invitrogen Co., Carlsbad, California, USA). Cells were then incubated with MSC from different donors in transwell conditions (TW) at three different HECV/MSC ratios (20:1, 10:1, and 4:1) for 24 hours. MSC were seeded in the upper chamber and HECV in the lower chamber. Before seeding HECV were irradiated at $5000 \mathrm{rad}$, washed once with PBS, counted, and plated with or without MSC.

\section{RNA isolation and quantitative real-time PCR}

Total RNA was isolated from either lymphocytes or endothelial cells using the Trizol reagent (Sigma Aldrich, Saint Louis, Missouri, USA) [42]. First-strand cDNA was synthesized in a Master-cycler apparatus (Eppendorf, Milan, Italy) from $1 \mu \mathrm{g}$ total RNA as described elsewhere [43]. Gene expression levels were quantified by quantitative real-time RT-PCR (qPCR) using Chromo4TMSystem PCR apparatus and iTaq SYBR Green Supermix (Biorad, Milan, Italy). Amplification reactions were performed in a final volume of $25 \mu \mathrm{l}$ containing $0.3 \mu \mathrm{M}$ each primer, 10 ng cDNA, 1× SybrGreen PCR Master Mix. All primers (Table 1) were designed ad hoc starting from the coding sequences available on the GenBank database (http://www.ncbi.nlm.nih.gov/Genbank/Genbank Search.html) and were synthesized by TibMolBiol custom oligosynthesis service. A melting curve of RT-PCR products $\left(55-94^{\circ} \mathrm{C}\right.$ ) was acquired to ensure the absence of artifacts. Relative expression of target mRNA was calculated using the comparative $\mathrm{Cq}$ method and was normalized for the expression of GAPDH gene [44]. The normalized expression was thus expressed as the relative quantity of mRNA (fold induction) with respect to controls (C).

\section{Flow cytometric analysis of lymphocyte surface antigens}

Cells were stained with the specific primary mAb for 30 minutes at $4{ }^{\circ} \mathrm{C}$, washed once with PBS, and analyzed. For coculture experiments, cells were additionally stained with Live Dead Fixable Near-IR Dead Cell-Stain Kit (Invitrogen) for 30 minutes at room temperature to exclude apoptotic cells by flow cytometric gating strategies (FSC-A vs. FL6-A dotplot). All immunolabeling procedures, unless otherwise indicated, were performed in the dark. The following mAbs were employed: CD34FITC, CD73PE, CD44FITC, CD14FITC, CD45FITC, CD45PE-Cy5, CD54 APC, CD54PE-Cy5 (BD Biosciences), CXCR3FITC and CXCR3APC (R\&D Systems), CD49d PE, CD90PE-Cy5, CD105APC, CD102PE, and CD106 APC (Biolegend Europe BV, London, UK), and KI67FITC (Dako Italia SpA, Milan, Italy). On the $\mathrm{CD}^{+}$lymphocyte population, the proportion of cells expressing $\alpha 4$ integrin, ICAM-1, and CXCR3 in the different experimental conditions was measured. On HECV, 
Table 1 Sequences of the primer pairs used for quantitative real-time RT-PCR analysis

\begin{tabular}{llll}
\hline Gene & $\begin{array}{l}\text { Accession } \\
\text { number }\end{array}$ & $\begin{array}{l}\text { Forward primer } \\
5^{\prime} \rightarrow 3^{\prime}\end{array}$ & $\begin{array}{l}\text { Reverse primer } \\
5^{\prime} \rightarrow 3^{\prime}\end{array}$ \\
\hline a4 integrin & NM_000885.4 & GGAATATC AGTTTTACACAAAGG GAG CCA GTC CAG TAA GAT GA \\
CXCR3 & NM_001114380.1 & GCTGTCCCCACAAAAAGTG & CCG GAA GGT CAC GTT GAA \\
ICAM-1 & NM_001142797.1 & CCAGCCATGGTCCTTGAG & GGG CCG TAC TTC CTC AAC T \\
IFNY & NM_000201.2 & CAACCGGAAGGTGTATGAAC & CGA GGT GTT CTC AAA CAG CTC \\
ALCAM & NM_000619.2 & GGCATTTGAAGAATTGGAAG & TाT GGA TGC TCT GGT CAT CTT \\
GAPDH & NM_001627.2 & CGT CTG CTC TTC TGC CTC TT & TAG GTG CCT CAA ACA CGT TG \\
\hline ALCAM aCTC TTG TGCT TGC TGG GGC TG
\end{tabular}

ALCAM activated leucocyte cell adhesion molecule, IFN $\gamma$ interferon gamma, ICAM intercellular adhesion molecule

we recorded the shift in the mean fluorescence intensity (MFI) for each adhesion molecule under the different experimental conditions. Moreover, production of IFNy by activated $\mathrm{CD}^{+}$lymphocytes was determined using Flow Cytomix particle-based assay (Biosciences, Prodotti Gianni, Milan, Italy), according to the manufacterer's instructions [45]. All flow cytometric analyses were performed by a FACS Canto flow cytometer (BD Biosciences) and data were collected and analyzed by DIVA software (BD Biosciences). Flow Cytomix particle-based assay data were analyzed with FlowCytomixPro 1.0 Software, eBioscience, San Diego, California, USA.

\section{$\mathrm{CD}^{+}$cell proliferation analysis}

Cell proliferation was measured by ${ }^{3} \mathrm{H}$-thymidine $\left({ }^{3} \mathrm{H}\right.$-TdR) incorporation. $\mathrm{CD}^{+}$cells cultured in the absence or in the presence of MSC in a transwell system were pulsed with $0.5 \mu \mathrm{Ci} /$ well ${ }^{3} \mathrm{H}-\mathrm{TdR}$ (5 Ci/mmole specific activity; GE Healthcare Europe $\mathrm{GmbH}$, Milan, Italy) for 8 hours. At the end of incubation, cells were harvested onto Multiscreen Harvest plates (Millipore, Billerica, MA, USA) using a 96well plate-automated cell harvester (Tomtec, Handem, CT, USA). Scintillation liquid (Fisher Chemicals, Leicester, UK) was then added and ${ }^{3} \mathrm{H}$-TdR incorporation was measured by liquid scintillation spectroscopy using a beta-counter (Chameleon TM 425-104 Multilabel Counter -Bioscan, Washington, USA). The results expressed in counts per minute $(\mathrm{kcpm}, \mathrm{cpm} \times 1000)$ are given as the mean value of triplicate wells. In the same experiments, $\mathrm{CD}^{+}$cells cocultured as already described were also analyzed by flow cytometry for Ki67 intranuclear expression to identify $\mathrm{KI}^{+} 7^{+}$ cycling T cells.

\section{$\mathrm{CD}^{+}$lymphocyte migration analysis}

Chemotaxis of $\mathrm{CD}^{+}$lymphocytes was investigated using 24-transwell plates with $5 \mu \mathrm{m}$ pore size polycarbonate membrane (Corning Costar, Celbio, Milan, Italy) as reported elsewhere [46]. $\mathrm{CD}^{+}$lymphocytes were grown for 48 hours with or without MSC (4:1 ratio) in the presence of $\alpha \mathrm{CD} 3(10 \mu \mathrm{g} / \mathrm{ml})$ and $\alpha \mathrm{CD} 28(1 \mu \mathrm{g} / \mathrm{ml})$ in a transwell system. Then, $5 \times 10^{5} \mathrm{CD}^{+}$lymphocytes were dispensed in the upper chamber, whereas $600 \mathrm{ng} / \mathrm{ml}$ CXCL10 (IP10; R\&D Systems) or medium alone was added to the lower chamber. Plates were incubated for 2 hours at $37{ }^{\circ} \mathrm{C}$, and then cells that migrated into the lower chamber were harvested and counted. Results were expressed as \% input, calculated as the \% ratio between the number of $\mathrm{CD}^{+}$cells dispensed in the upper chamber and that of cells recovered from the lower chamber after migration. Net \% input (namely the difference between the input obtained following chemokine stimulation and that obtained with medium alone) was used for statistical analysis of the results.

\section{Statistical analysis}

Data of qPCR are expressed as mean \pm standard deviation (SD) of two independent RNA extractions from at least five independent experiments, and each PCR was performed in quadruplicate. Data of flow cytometry are expressed as mean \pm SD of the frequency of populations measured in at least five independent experiments. Statistical significance was determined by analysis of variance followed by the Bonferroni post-hoc test (Instat software; GraphPad Software, Inc., San Diego, CA, USA). The Mann-Whitney $U$ test was used for chemotaxis-related experiments.

\section{Results}

\section{MSC inhibit T-cell proliferation and function}

In preliminary experiments, activated PBMC were cocultured with MSC at different PBMC/MSC ratios (20:1, 10:1, and 4:1) using both the cell-to-cell contact (CC) and TW in order to select the experimental model to be used in the study. When IFNy expression was assessed by qPCR, a significant upregulation was observed in activated PBMC with respect to controls (19-fold; $p<0.001$ ) (Fig. 1a). In the CC condition (Fig. 1a), MSC led to a dose-dependent reduction in IFN $\gamma$ expression with a decrease of $-94 \%$ with respect to activated cells $(p<0.001)$ in the presence of the highest MSC dose (PBMC/MSC 


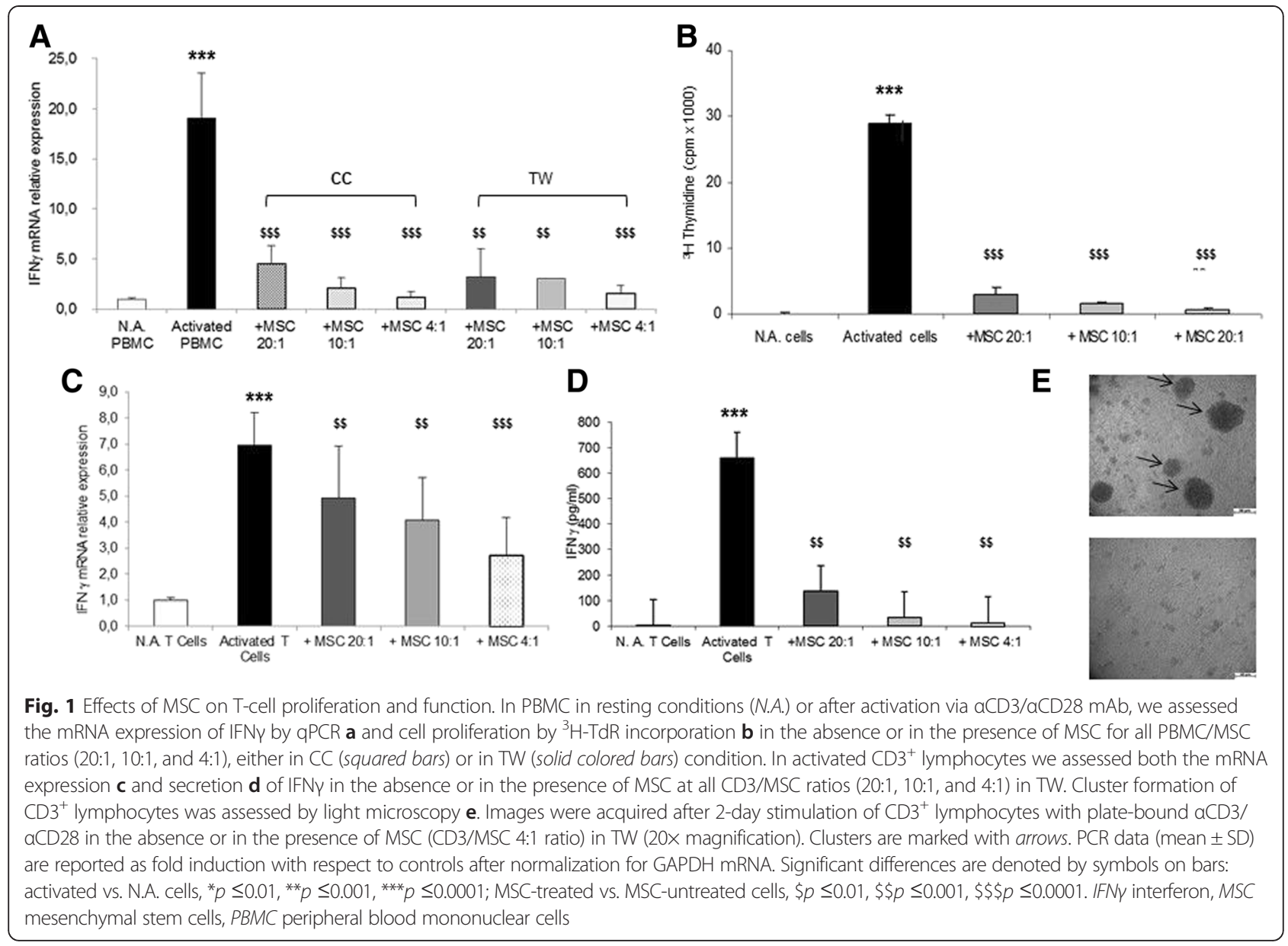

ratio $4: 1)$, and of about $-80 \%(p<0.001)$ for the other ratios. Similar effects were observed in TW (Fig. 1a), where the maximum reduction in IFN $\gamma$ mRNA level $(-92 \%$ with respect to activated cells; $p<0.001)$ was observed at the 4:1 PBMC/MSC ratio, but a significant decrease of about $-83 \%(p<0.01)$ was observed also at 20:1 and 10:1 ratios. As these data clearly indicate that MSC effects on PBMC do not depend on $\mathrm{CC}$, we used TW to test the possible effects of MSC on PBMC proliferation by using ${ }^{3} \mathrm{H}-\mathrm{TdR}$ incorporation (Fig. 1b). PBMC consistently proliferated upon in vitro activation (mean $0.11 \pm 0.03$ vs. $29.02 \pm 1.31$, unstimulated vs. stimulated, respectively; $p<0.001$ ). A dose-dependent inhibition of cell proliferation was observed when cells were cocultured with MSC at all PBMC/MSC ratios for 4 days (means $3.05 \pm 1.07$, $1.68 \pm 0.14$, and $0.67 \pm 0.18$ for $20: 1,10: 1$, and $4: 1$ ratios, respectively; $p<0.001$ ). Correspondingly, most PBMC cultured in the absence of MSC constitutively expressed Ki67, a classical marker of cell proliferation, and its expression strongly decreased in the presence of MSC in a dose-dependent manner (data not shown).
Using TW, expression and secretion of IFNY was measured in selected $\mathrm{CD}^{+}$lymphocytes cocultured with MSC in TW (Fig. 1c). After activation, $\mathrm{CD}^{+}$cells showed a marked upregulation of IFN $\gamma$ expression (about sevenfold with respect to control; $p<0.001$ ) that was significantly reduced in the presence of MSC. The maximum reduction (-67\% with respect to activated cells; $p<0.001$ ) was observed at the highest MSC dose (CD3/MSC ratio 4:1), but a significant decrease of about $-33 \%(p<0.01)$ was observed also at 20:1 and 10:1 ratios (Fig. 1c). On the same samples, the secretion of IFNY in the medium was evaluated using flow cytometry (Fig. 1d). The IFN $\gamma$ level increased in activated $\mathrm{CD}^{+}$cells $(141$-fold with respect to resting cells; $p<0.001$ ) and decreased in a dosedependent manner when $\mathrm{CD}^{+}$cells were cocultured with MSC at all CD3/MSC ratios $(p<0.01)$ (Fig. 1c).

Finally, the ability of $\mathrm{CD}^{+}$cells to form cluster was assessed by light optical microscopy (Fig. 1e, upper and lower). According to expectations, $\mathrm{CD}^{+}$cell clusters were observed upon stimulation with $\alpha C D 3 / \alpha C D 28$ (Fig. 1e, upper), while the presence of MSC in coculture with $\mathrm{CD}^{+}$cells prevented cluster formation (Fig. 1e, lower). 


\section{MSC inhibit expression of adhesion molecules on $\mathrm{CD}^{+}$ lymphocytes}

At first, the basal expression of the adhesion molecules under analysis was assessed in resting $\mathrm{CD}^{+}$lymphocytes. While $\alpha 4$ integrin mRNA was expressed in basal conditions, both ICAM-1 and $\beta 2$ integrin were barely detectable (Cq values of about 25 for $\alpha 4$ integrin, and 32 for ICAM-1 and $\beta 2$ integrin) (data not shown).

At mRNA level, $\alpha 4$ integrin expression was upregulated upon activation with respect to resting $\mathrm{CD}^{+}$lymphocytes (2.7-fold induction; $p<0.001$ ) (Fig. 2a). A significant reduction with respect to activated cells $(-57 \% ; p<0.001)$ was observed with the highest MSC dose in TW (4:1 CD3/MSC ratio); an evident but not significant effect could be appreciated also at 20:1 and 10:1 ratios. Activated $\mathrm{CD}^{+}$cells showed also a significant increase in mRNA expression of ICAM-1 with respect to resting lymphocytes (7.1-fold induction; $p<0.001$ ) (Fig. 2b). Also in this case the highest MSC dose significantly reduced ICAM-1 expression with respect to activated $\mathrm{CD}^{+}$ cells $(-86 \% ; p<0.001)$ (Fig. 2b). For comparison, similar experiments were performed on PBMC cocultured with MSC at different PBMC/MSC ratios (20:1, 10:1, and 4:1) using both CC and TW, and similar inhibitory effects were observed in both culture conditions (data not shown).

Although activated $\mathrm{CD}^{+}$cells did not show any significant increment in mRNA level of $\beta 2$ integrin with respect to resting cells, a significant reduction of about $-67 \%$ $(p<0.001)$ was observed for all the CD3/MSC ratios without a dose dependence (Table 2).

Flow cytometry studies performed in parallel showed that $\alpha 4$ integrin was constitutively expressed at the plasma membrane level on resting $\mathrm{CD}^{+}$lymphocytes (mean $52.25 \pm 13.87$ ) (Fig. 2c), while very low expression (mean $2.76 \pm 1.98$ ) was observed for ICAM-1 (Fig. 2 d), in accordance with qPCR data. Upon in vitro activation, $\mathrm{CD}^{+}$cells showed a slight increase in the proportion of $\alpha 4$ integrin (mean 66.81 $\pm 19.7, p<0.01$ ), and a marked increase in the proportion of ICAM-1 (mean 94.5 $\pm 3.47, p<0.001$ ) with respect to resting cells. The presence of MSC in TW slightly reduced the proportion of $\alpha 4$ integrin on

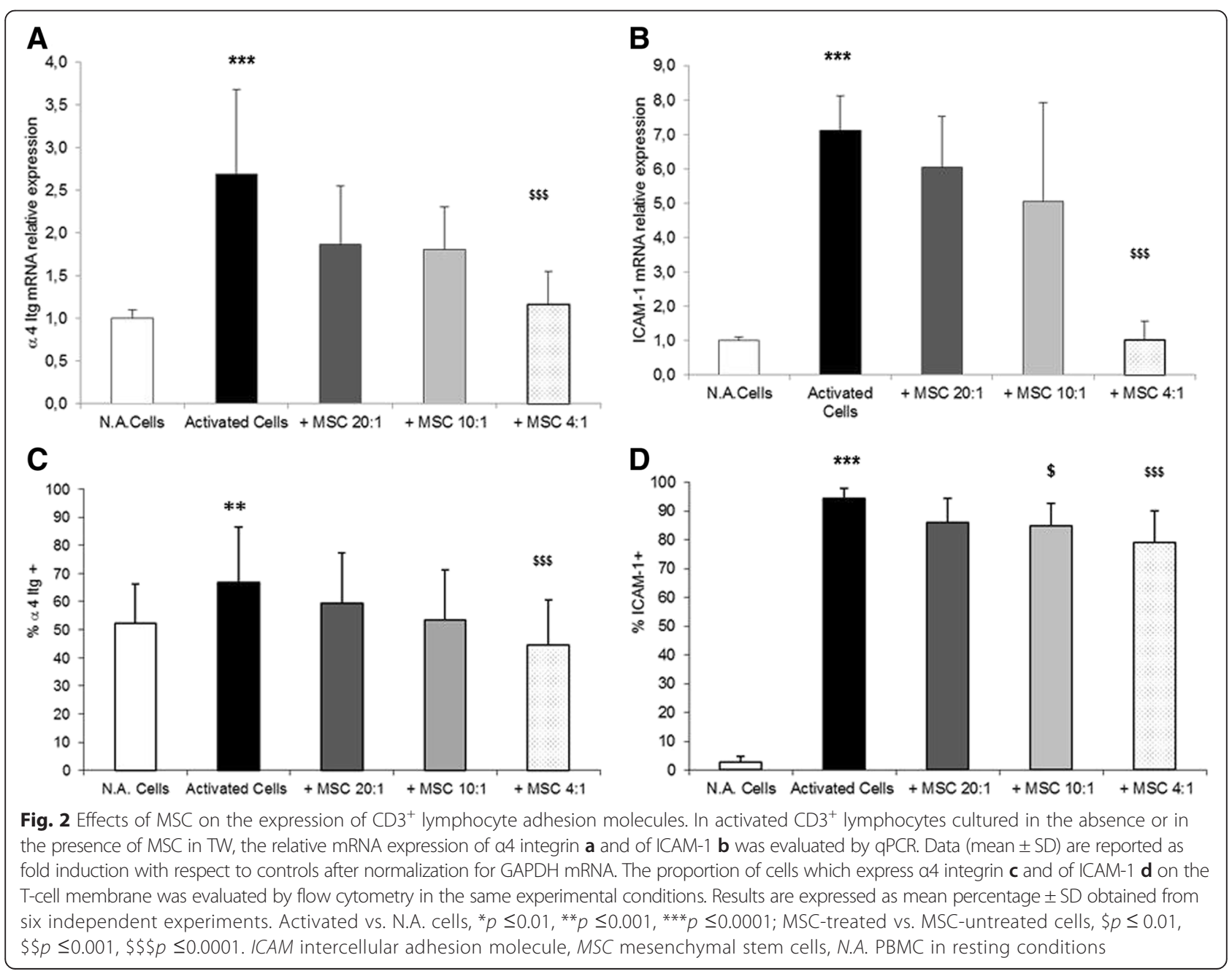


Table 2 Relative expression of $\beta 2$ integrin mRNA in activated $\mathrm{CD}^{+}$lymphocytes cultured in the absence or in the presence of MSC at three CD3/MSC ratios (20:1, 10:1, and 4:1)

\begin{tabular}{lc}
\hline Treatments & Fold induction \\
\hline Activated $\mathrm{CD}^{+}$cells & $0.87 \pm 0.28$ \\
Activated $\mathrm{CD}^{+}$cells + MSC 20:1 & $0.29 \pm 0.33^{* *}$ \\
Activated $\mathrm{CD}^{+}$cells + MSC 10:1 & $0.30 \pm 0.35^{* *}$ \\
Activated $\mathrm{CD}^{+}$cells + MSC 4:1 & $0.28 \pm 0.18^{* * *}$ \\
\hline mRNA expression was evaluated by qPCR, and values representing the mean \\
\pm SD of three different experiments are reported as fold induction with respect \\
to controls (no activated CD3 ${ }^{+}$lymphocytes) after normalization for GAPDH \\
mRNA. Significant differences: MSC-treated cells vs. activated cells, ${ }^{* *} p \leq 0.01$, \\
$\begin{array}{l}* * * \\
\text { MSC mesenchymal stem cells }\end{array}$
\end{tabular}

the $\mathrm{CD}^{+}$surface with respect to activated cells $(-7 \%$ for $20: 1$ ratio, $-13 \%$ for $10: 1$ ratio, and $-22 \%$ for $4: 1$ ratio), but the statistical significance was reached only with the highest dose of MSC $(p<0.001)$ (Fig. 2c). The presence of MSC markedly reduced also the proportion of ICAM-1 on the cell membrane in a dose-dependent manner $(-9.7 \%$ for $10: 1$ ratio and $-15.4 \%$ for $4: 1$ ratio; $p<0.05$ and $p<0.001$, respectively) (Fig. 2d). No differences were observed for $\beta 2$ integrin upon exposure to MSC at different ratios (data not shown).

\section{Inhibition of CXCR3 expression by MSC reduced CXCL10- dependent chemotaxis of $T$ cells}

We observed that CXCR3 was constitutively expressed in resting $\mathrm{CD}^{+}$cells, and its transcription increased significantly (Fig. 3a) upon activation with $\alpha \mathrm{CD} 3 / \alpha \mathrm{CD} 28$ (5.6fold induction with respect to resting cells; $p<0.001$ ). This change was still detectable at plasma membrane level (Fig. 3b), although the increase in the CXCR3 proportion on the cell membrane did not reach significance (means $37.1+8.58$ vs. $45.13 \pm 10.21$ ). The upregulation of CXCR3 expression was prevented by the presence of MSC in a dose-dependent manner. Both the 10:1 and 4:1 CD3/MSC ratios were effective on preventing the upregulation of CXCR3, which showed a significant reduction of $-50 \%$ and $-80 \%$, respectively, with respect to activated cells $(p<0.001)$ (Fig. 3a). These effects were evident also at plasma membrane level, where the presence of MSC led to a dose-dependent reduction in the proportion of CXCR3 on the plasma membrane $(-28.1 \%$ for $20: 1$

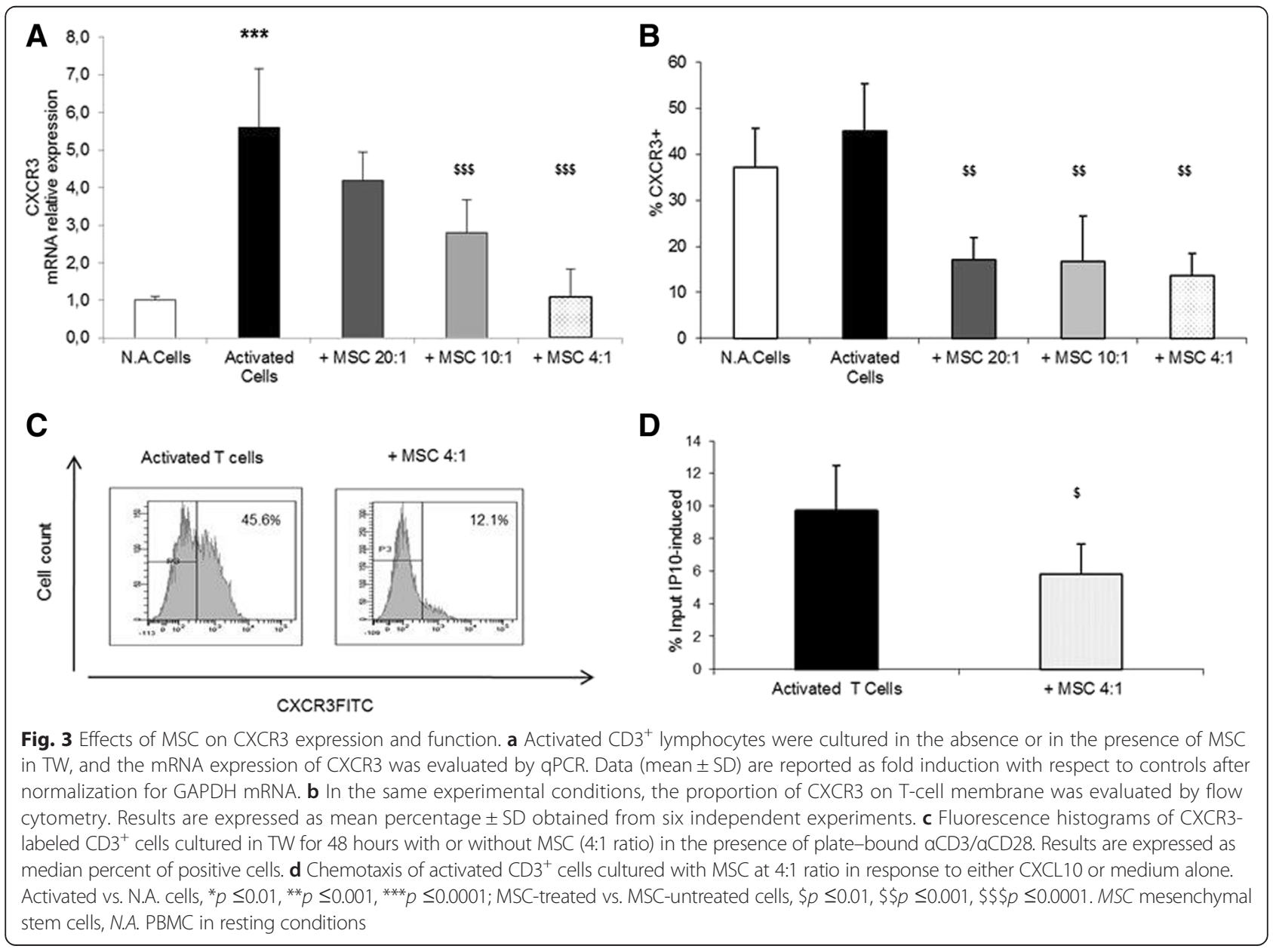


ratio, $-28.4 \%$ for $10: 1$ ratio, and $-31.5 \%$ for $4: 1$ ratio; $p<0.001$ ) (Fig. 3b).

In order to explore whether the effects of MSC reflect on the CXCR3-related T-cell function, the chemotactic activity of activated $\mathrm{CD}^{+}$cells was assessed. $\mathrm{CD}^{+}$cells were cultured for 2 days in the absence or in the presence of MSC in TW (CD3/MSC 4:1 ratio). Then, the expression of CXCR3 and the response to CXCL10, the selective ligand of CXCR3, were evaluated. We found a marked decrease in CXCR3 expression on the surface of $\mathrm{CD}^{+}$cell cultured with MSC with respect to activated cells (means $48.36 \pm 10.03$ vs. $22.44 \pm 11.84 ; p<0.01$ ) that was paralleled by a significant $(p<0.05)$ inhibition of CXCL-10-driven chemotaxis (Fig. 3c, d).

\section{MSC inhibit expression of adhesion molecules on HECV}

The possible effects of MSC on endothelial cells were assessed focusing on some adhesion molecules relevant for their interaction with $\mathrm{T}$ cells. HECV constitutively express ALCAM and ICAM-1 at both mRNA and membrane level $($ MFI $=1927 \pm 488.88$ and MFI $=7311+1398$, respectively) (Fig. 4), whereas no constitutive expression of either V-CAM-1 or ICAM-2 was observed both at the mRNA and the membrane level (data not shown).

Upon stimulation with IFN $\gamma$, HECV showed a marked increase in ICAM-1 expression at both the mRNA (10.1fold induction with respect to controls; $p<0.001$ ) and the plasma membrane (mean 14,599 $\pm 1635.42, p<0.001$ ) levels (Fig. 4a, b, respectively). Also ALCAM expression was upregulated by IFN $\gamma$ at the mRNA level (1.8-fold induction; $p<0.01$ ), but the increase was not significant at the membrane level (mean $=2406 \pm 520.99$ ) (Fig. 4c, d, respectively). The upregulation of ICAM-1 mRNA expression was reduced by the presence of MSC at all HECV/ MSC ratios $(-33 \%,-59 \%$, and $-68 \%$ for $201: 1,10: 1$, and 4.1 ratios, respectively, with respect to activated cells; $p<0.001$ ) (Fig. 4a). Also, at the plasma membrane level, MSC reduced ICAM-1 with respect to stimulated cells $($ MFI $=14,599$ for stimulated HECV vs. MFI $=-2517$, MFI $=-2624$, and MFI $=-2288$ for 20:1, 10:1, and 4:1 HECV/MSC ratios, respectively; $p<0.01$ ) (Fig. $4 \mathrm{~b}$ ). The upregulation of ALCAM expression was prevented by the presence of MSC at all HECV/MSC ratios both at the mRNA (about $-55 \%$ for all ratios with respect to stimulated cells) (Fig. 4c) and at the plasma membrane level $(\mathrm{MFI}=2406$ for stimulated HECV vs. MFI $=-711$, MFI $=-726$, and MFI $=-504$ for 20:1, 10:1, and 4.1 HECV/MSC ratios, respectively; $p<0.01$ ); a slight decrease was observed at the highest HECV/MSC 4:1 ratio; $p<0.05$ (Fig. 4d).

\section{Discussion}

In this study, we demonstrated that MSC significantly act on adhesion molecules and receptors localized on both T- lymphocyte and endothelial cell membrane whose interactions are involved in lymphocyte extravasation and trafficking across the BBB. T-cell homing into the CNS is very low in physiological conditions, but it remarkably increases under inflammatory cues such those occurring in $\mathrm{EAE}$ and $\mathrm{MS}$ when encephalitogenic $\mathrm{CD} 4^{+}$and $\mathrm{CD} 8^{+}$cells infiltrate the CNS [1]. The process of leukocyte extravasation consists of a finely regulated sequence of steps that is mediated by different classes of surface molecules sustaining the interaction between leukocytes and endothelial cells $[3,47]$. The initial contact of activated lymphocytes with the BBB endothelium is mediated by selectins [3], while integrins trigger the subsequent firm adhesion [11, 12], and then the interaction between ICAM-1 on T cells and ALCAM on endothelial cells led to the final diapedesis $[21,22]$.

With regards to therapy of MS and other autoimmune diseases, the ultimate goals are twofold: to eliminate/ reduce the self-reactive lymphocytes halting the immune attack to the CNS; and to attempt repair of the existing damage. Many studies indicated that transplantation of MSC is of functional benefit in animal models of MS [33, 34]. Different mechanisms have been suggested including induction of immune tolerance toward myelin antigens, release of trophic and anti-apoptotic factors, and induction of endogenous neurogenesis [48]. Interestingly, MSC decrease T-cell infiltration in the CNS of mice with EAE [33] and impair the encephalitogenic potential of $\mathrm{T}$ cells [34]. Based on the results presented in this manuscript, we provide a significant insight into the possible molecular mechanism impairing T-cell extravasation under inflammatory conditions as mimicked by the exposure of both $\mathrm{T}$ cells and endothelial cells to IFN $\gamma$. Although these in vitro results should be cautiously considered as validation of the in vivo mechanisms of action of administered MSC and by no means as an indication of optimal yields of cells to be transplanted, they are a solid "proof of concept" permitting to elucidate the possible interactions between MSC, immune cells, and endothelial cells, which might be relevant for some clinical conditions such as MS.

At first, we observed that, in vitro, MSC inhibit IFN $\gamma$ expression by PBMC upon $\alpha \mathrm{CD} 3 / \alpha C D 28$ stimulation using both CC and TW. This clearly indicates that such an effect does not depend on a physical contact between MSC and PBMC, but on soluble factors released by MSC. Moreover, MSC play a profound and dose-dependent inhibition of PBMC proliferation and, interestingly, MSC presence blocks $P B M C$ in $G_{0} / G_{1}$ phases of the cell cycle as shown by the decrease in expression of KI67 (data not shown), in accordance with previous reports $[28,49]$. On the other hand, MSC exerted the same inibitory effects on selected $\mathrm{CD}^{+}$ lymphocytes. 


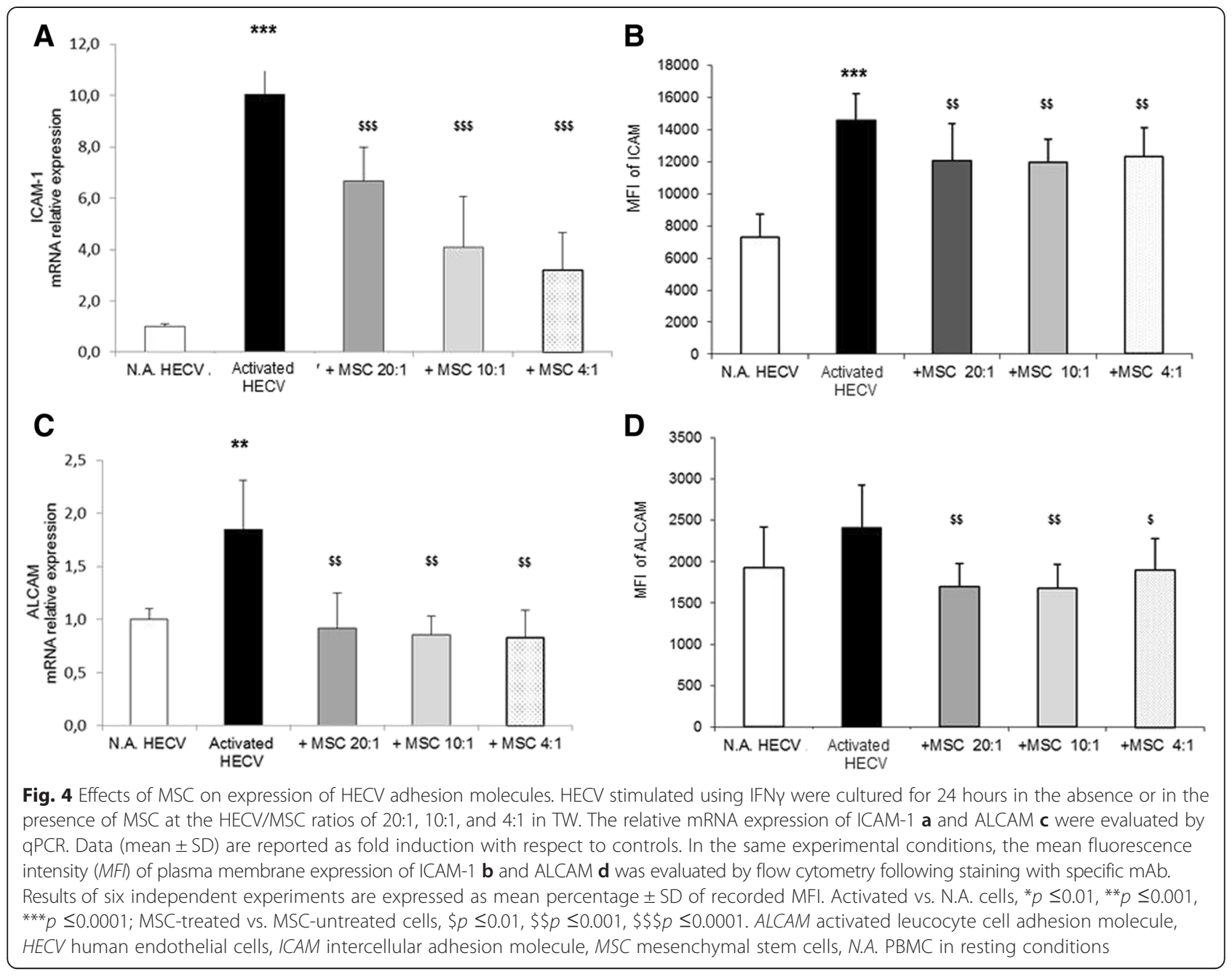

In the light of their effects on activation of $\mathrm{CD}^{+}$lymphocytes, we investigated whether MSC act on the pool of molecules involved in T-cell trafficking into the CNS such as $\alpha 4$ integrin, $\beta 2$ integrin, ICAM- 1 , and CXCR3. We found a decrease in the level of $\alpha 4$ integrin upon MSC exposure. In particular, we observed that activated $\mathrm{T}$ cells overexpressed $\alpha 4$ integrin and this upregulation was prevented by the presence of MSC in TW. This effect of MSC on activated T cells in vitro resembles the effects played by the mAb NTZ in vivo [50]. NTZ is "the first of a new class of drugs known as elective adhesion molecule inhibitor" employed to treat relapsingremitting MS [5], and Harrer et al. [50] described a reduction in both $\alpha 4$ and $\beta 1$ levels on T-cell, B-cell, and natural killer cell membrane in response to NTZ therapy in MS patients. Our results suggest that MSC may mimic the effect of NTZ on $\alpha 4$ integrin expression in T cells, and this could represent one of the molecular mechanisms supporting the clinical efficacy of MSC transplantation in the animal model of MS. By contrast, $\beta 2$ integrin, the constituent of LFA-1 antigen required for a broad range of leukocyte actions, was not upregulated in stimulated $\mathrm{CD}^{+}$cells, in accordance with previous data showing that activation of $\mathrm{T}$ cells via the $\mathrm{T}$-cell receptor (TCR) did not change LFA-1 expression [51]. However, MSC are able to reduce the mRNA level of $\beta 2$ integrin and this action could be synergic with the inhibitory effect on $\alpha 4$ integrin possibly leading to a strengthening of the inhibition of T-cell extravasation.

Activation of $\mathrm{CD}^{+}$cells also led to an increased expression of CXCR3, a key receptor of Th1 lymphocytes, and this upregulation was inhibited by the presence of MSC in a dose-dependent manner. It has to be noted that $\mathrm{T}$ lymphocytes isolated from the cerebrospinal fluid (CSF) of MS patients express high levels of CXCR3 [19] and that $\mathrm{CXCR}^{+}{ }^{+} \mathrm{T}$ cells are present in the infiltrates observed in MS tissues [19]. Here, we demonstrated that not only expression of CXCR3 on $\mathrm{CD}^{+}$lymphocytes, but also their ability to migrate under the chemotactic stimulus of CXCL10, which has a relevant role in the pathophysiology of MS [52], is inhibited by MSC. This result is of particular interest because it indicates that 
MSC also inhibit the effector function of T cells, in contrast to a previous study showing that the inhibitory effect of MSC was confined to proliferation of $\mathrm{T}$ cells rather than to their effector functions [53]. Taken together, our data suggest that the inhibition exerted by MSC on CXCR3 expression might cooperate with the other changes induced on het $\mathrm{CD}^{+}$cell surface to prevent intrathecal accumulation of T cells in MS.

In line with our finding, an earlier report stated that blocking CXCR3 function was associated with less severe EAE and diminished T-cell infiltration of the CNS [54], although other studies reported that $\mathrm{CXCR}^{-/-}$mice develop more severe EAE [55] and that CXCR3 signaling has a protective role by retaining the $\mathrm{T}$ cells in the perivascular compartment [56].

Similar suppressive action of MSC was observed on ICAM-1 expression. ICAM-1 is constitutively expressed at low levels on $\mathrm{T}$ cells and is strongly upregulated following stimulation. By contrast, ICAM-1 expression is high in the endothelial cells and is further increased upon stimulation with IFN $\gamma$. Here, we demonstrated that MSC modulate ICAM-1 expression on both T cells and endothelial cells by decreasing the frequency of $\mathrm{CD}^{+}$cells expressing ICAM-1, and the antigen density on HECV. Such an effect is relevant since it indicates a stronger immunomodulation effect of MSC through a bidirectional action that, on one side, blocks ICAM-1 expression on the T-lymphocyte surface and, on the other side, decreases ICAM-1 expression in the endothelial cell membrane. This effect may be considered protective, despite previous controversial results. In fact, some reports showed that blocking of ICAM-1 led to a protective outcome in EAE $[4,57]$, whereas other studies reported no protection or even increased disease severity $[58,59]$. On the other hand, an attenuated disease was observed in ICAM-1 null mutant mice (ICAM-1 ${ }^{\text {null }}$ ), which are deficient in all ICAM-1 isoforms [60]. However, we must not forget that blocking of ICAM-1 in vivo affects both leukocyte and endothelial cells, and previous studies showed that the role of ICAM-1 in EAE development was linked to its expression on both endothelial cells and T lymphocytes [61, 62].

When we investigated the possible cross-talk between MSC and endothelial cells, we observed that MSC in coculture with $\mathrm{HECV}$ decreased IFN $\gamma$-dependent upregulation of ALCAM, an adhesion molecule expressed on endothelial cells [63]. Interestingly, in both MS and EAE, ALCAM expression at the BBB level is upregulated in active lesions [23]. Our finding therefore suggests that suppressive action of MSC on T-cell infiltration is strengthened by their effect on adhesion molecules localized on endothelial cells.

Taken together, our results indicate that MSC act directly on both $\mathrm{T}$ lymphocytes and endothelial cells by reducing the expression of those surface molecules highly expressed upon inflammatory stimuli playing a role in the
T-cell-endothelial cell interaction at the BBB, thus possibly affecting the consequent lymphocyte entry into CNS. Although these results have been exclusively obtained in vitro, the ability of MSC to decrease the expression of key molecules involved in the migration of $\mathrm{T}$ cells into the CNS reinforces their therapeutic efficacy in treating MS [48]. Moreover, changes induced on the lymphocyte surface are strengthened by the inhibition of T-cell proliferation and of the chemokine-driven chemotaxis. All of these events may act in synergy and could explain the observation of a reduced number of $\mathrm{T}$ cells infiltrating the CNS upon MSC administration [33]. By using different coculture systems we have also clarified that the action of MSC mainly occurs through the release of soluble factors and does not require physical contact between lymphocytes and endothelial cells. Interestingly, these soluble factors are not constitutively secreted by MSC but only under the condition of cross-talk with activated lymphocytes. In fact, when lymphocytes were incubated in MSC-conditioned medium-that is, medium containing soluble factors secreted by MSC in basal conditions without coculture with PBMC-no effects were observed at the level of the surface molecules under analysis (data not shown).

In conclusion, although MSC are described as cells having high differentiation potential, their effector functions seem to be based less on in situ transdifferentiation or fusion ability, and more on paracrine effects and crosstalk with other cells present within diseased tissues. In particular, MSC might exert their therapeutic effects also through the inhibition of extravasation of T cells, an event of high relevance for the treatment of MS.

\section{Conclusions}

The results of this study will enhance our knowledge about the mechanisms sustaining the immunosuppressive action of MSC by focusing on the interaction between MSC and endothelial cells. Our findings indicate that the immunosuppressive effect of MSC does not exclusively depend on their anti-proliferative activity on $\mathrm{T}$ cells, but also on the impairment of leukocyte migration through the BBB by acting on those adhesion molecules and receptors that are responsible for T-cell rolling.

\footnotetext{
Abbreviations

ALCAM: Activated leucocyte cell adhesion molecule; BBB: Blood-brain barrier; CC: cell-to-cell contact; CNS: Central nervous system; CSF: cerebrospinal fluid; DMEM: Dulbecco's modified Eagle's medium; EAE: Experimental autoimmune encephalomyelitis; EDTA: Ethylenediamine tetraacetic acid; FCS: Fetal calf serum; GvHD: Graft versus host disease; ${ }^{3} \mathrm{H}-\mathrm{TdR}$ : ${ }^{3} \mathrm{H}$-thymidine; HECV: Human endothelial cell; HSC: Hematopoietic stem cells; ICAM: Intercellular adhesion molecule; IFNy: Interferon gamma; IgSF: Imunoglobulin superfamily; LFA-1: Lymphocyte function-associated antigen-1; mAB: Monoclonal antibody; MFI: Mean fluorescence intensity; MS: Multiple sclerosis; MSC: Mesenchymal stem cells; NTZ: Natalizumab; PBMC: Peripheral blood mononuclear cells; PBS: Phosphate-buffered saline; qPCR: Quantitative real-time RT-PCR; SD: Standard deviation; TCR: T-cell receptor; Th17: T helper 17 cells; Th1: Type 1 helper cells; TW: Transwell conditions; VLA-4: Very late antigen-4.
} 


\section{Competing interests}

The authors declare that they have no competing interests.

\section{Authors' contributions}

FB performed cell cultures and the immunological and flow cytometry assays, analyzed the data, and drafted the manuscript. EC performed the qPCR experiments, analyzed the data, and revised the manuscript. FG provided the bone marrow samples, reviewed the data, and revised the manuscript. AV participated in the study design, reviewed the data, and revised the manuscript. GM participated in the study design, reviewed the data, and revised the manuscript. AU designed the study, reviewed the data, and revised the manuscript. LV designed the study, analyzed the data, and wrote the manuscript. All authors read and approved the manuscript.

\section{Acknowledgements}

This research was supported by grants to LV from University of Genova and from Compagnia San Paolo (Torino), and by grants to AU from the Fondazione Italiana Sclerosi Multipla and the "Progetto MESEMS", Mesenchymal Stem Cells for Multiple Sclerosis Project.

\section{Author details}

'Department of Neurology, Rehabilitation, Ophthalmology, Genetics, Maternal and Child Health (DINOGMI), University of Genoa, IRCCS-AUO San Martino-IST, Largo Paolo Daneo 3, 16132 Genova, Italy. ${ }^{2}$ Centre of Excellence for Biomedical Research (CEBR), University of Genoa, Viale Benedetto XV 7 , 16132 Genova, Italy. ${ }^{3}$ Department of Earth, Environment and Life Sciences (DISTAV), University of Genoa, Corso Europa 26, 16132 Genova, Italy. ${ }^{4}$ Division of Hematology and Bone Marrow Transplant Unit, IRCCS-AUO San Martino-IST, Largo Rosanna Benzi 10, 16132 Genova, Italy.

\section{Received: 9 September 2015 Revised: 29 October 2015}

Accepted: 2 November 2015

Published online: 10 December 2015

\section{References}

1. Bartholomäus I, Kawakami N, Odoardi F, Schläger C, Miljkovic D, Ellwart JW, et al. Effector T cell interactions with meningeal vascular structures in nascent autoimmune CNS lesions. Nature. 2009;462(7269):94-8.

2. Lassmann $H$, Bruck W, Lucchinetti CF. The immunopathology of multiple sclerosis: an overview. Brain Pathol. 2007;17(2):210-8.

3. Engelhardt B. The blood-central nervous system barriers actively control immune cell entry into the central nervous system. Curr Pharm Des. 2008;14(16):1555-65

4. Archelos JJ, Jung S, Mäurer M, Schmied M, Lassmann H, Tamatani T, et al. Inhibition of experimental autoimmune encephalomyelitis by an antibody to the intercellular adhesion molecule ICAM-1. Ann Neurol. 1993;34(2):145-54.

5. Yednock TA, Cannon C, Fritz LC, Sanchez-Madrid F, Steinman L, Karin N. Prevention of experimental autoimmune encephalomyelitis by antibodies against alpha 4 beta1 integrin. Nature. 1992;356(6364):3-6.

6. Miller DH, Khan OA, Sheremata WA, Blumhardt LD, Rice GP, Libonati MA, et al. A controlled trial of natalizumab for relapsing remitting multiple sclerosis. N Engl J Med. 2003;348:15-23.

7. Hynes RO. Integrins: versatility, modulation, and signaling in cell adhesion. Cell. 1992;69(1):11-25.

8. von Andrian UH, Engelhardt B. Alpha4 integrins as therapeutic targets in autoimmune disease. N Engl J Med. 2003;348(1):68-72.

9. Archelos JJ, Previtali SC, Hartung HP. The role of integrins in immune-mediated diseases of the central nervous system. Trends Neurosci. 1999;22(1):30-8.

10. Baron JL, Madri JA, Ruddle NH, Hashim G, Janeway Jr CA. Surface expression of alpha 4 integrin by CD4 T cells is required for their entry into brain parenchyma. J Exp Med. 1993;177(1):57-68.

11. Engelhardt B. Molecular mechanisms involved in T cell migration across the blood-brain barrier. J Neural Transm. 2006;113(4):477-85.

12. Steinman L. Blocking adhesion molecules as therapy for multiple sclerosis: natalizumab. Nat Rev Drug Discov. 2005;4(6):510-8.

13. van de Stolpe A, van der Saag PT. Intercellular adhesion molecule-1. J Mol Med (Berl). 1996;74(1):13-33.

14. Steffen BJ, Butcher EC, Engelhardt B. Evidence for involvement of ICAM-1 and VCAM-1 in lymphocyte interaction with endothelium in experimental autoimmune encephalomyelitis in the central nervous system in the SJL/J mouse. Am J Pathol. 1994;145(1):189-201.
15. Greenwood J, Amos CL, Walters CE, Couraud PO, Lyck R, Engelhardt B. Intracellular domain of brain endothelial intercellular adhesion molecule-1 is essential for T lymphocyte-mediated signaling and migration. J Immunol. 2003;171(4):2099-108.

16. Reiss $Y$, Hoch G, Deutsch U, Engelhardt B. T cell interaction with ICAM-1-deficient endothelium in vitro: essential role for ICAM-1 and ICAM-2 in transendothelial migration of T cells. Eur J Immunol. 1998;28(10):3086-99.

17. Kebir H, Ifergan I, Alvarez Jl, Bernard M, Poirier J, Arbour N, et al. Preferential recruitment of interferon-gamma-expressing TH17 cells in multiple sclerosis. Ann Neurol. 2009;66(3):390-402.

18. Giunti D, Borsellino G, Benelli R, Marchese M, Capello E, Valle MT, et al. Phenotypic and functional analysis of T cells homing into the CSF of subjects with inflammatory diseases of the CNS. J Leukoc Biol. 2003;73(5):584-90.

19. Sørensen $T L$, Tani M, Jensen J, Pierce V, Lucchinetti C, Folcik VA, et al. Expression of specific chemokines and chemokine receptors in the central nervous system of multiple sclerosis patients. J Clin Invest. 1999;103(6):807-15.

20. García-López MA, Sánchez-Madrid F, Rodríguez-Frade JM, Mellado M, Acevedo A, García MI, et al. CXCR3 chemokine receptor distribution in normal and inflamed tissues: expression on activated lymphocytes, endothelial cells, and dendritic cells. Lab Invest. 2001;81(3):409-18.

21. Lee SJ, Benveniste EN. Adhesion molecule expression and regulation on cells of the central nervous system. J Neuroimmunol. 1999;98(2):77-88.

22. Lee BP, Imhof BA. Lymphocyte transmigration in the brain: a new way of thinking. Nat Immunol. 2008;9(2):117-8.

23. Cayrol R, Wosik K, Berard JL, Dodelet-Devillers A, Ifergan I, Kebir H, et al. Activated leukocyte cell adhesion molecule promotes leukocyte trafficking into the central nervous system. Nat Immunol. 2008;9(2):137-45.

24. Wagner M, Bilinska M, Pokryszko-Dragan A, Sobczynski M, Cyrul M, Kusnierczyk $P$, et al. ALCAM and CD6-multiple sclerosis risk factors. J Neuroimmunol. 2014;276(1-2):98-103.

25. da Silva Meirelles L, Chagastelles PC, Nardi NB. Mesenchymal stem cells reside in virtually all post-natal organs and tissues. J Cell Sci. 1996;11:2204-13.

26. Aggarwal S, Pittenger MF. Human mesenchymal stem cells modulate allogeneic immune cell responses. Blood. 2005;105(4):1815-22.

27. Uccelli A, Moretta L, Pistoia V. Mesenchymal stem cells in health and disease. Nat Rev Immunol. 2008;8(9):726-36.

28. Benvenuto F, Ferrari S, Gerdoni E, Gualandi F, Frassoni F, Pistoia V, et al. Human mesenchymal stem cells promote survival of $T$ cells in a quiescent state. Stem Cells. 2007;25(7):1753-60.

29. Crigler L, Robey RC, Asawachaicharn A, Gaupp D, Phinney DG, Robinson AP, et al. Human mesenchymal stem cell subpopulations express a variety of neuro-regulatory molecules and promote neuronal cell survival and neuritogenesis. Exp Neurol. 2006;198(1):54-64.

30. Ohtaki H, Ylostalo JH, Foraker JE, Robinson AP, Reger RL, Shioda S, et al. Stem/progenitor cells from bone marrow decrease neuronal death in global ischemia by modulation of inflammatory/immune responses. Proc Natl Acad Sci U S A. 2008;105(38):14638-43.

31. Lanza C, Morando S, Voci A, Canesi L, Principato MC, Serpero LD, et al. Neuroprotective mesenchymal stem cells are endowed with a potent antioxidant effect in vivo. J Neurochem. 2009;110(5):1674-84.

32. Uccelli A. Mesenchymal stem cells exert a remarkable regenerative effect requiring minimal CNS integration: commentary on: "Mesenchymal stem cells protect CNS neurons against glutamate excitotoxicity by inhibiting glutamate receptor expression and function" by Voulgari-Kokota et al. Exp Neurol. 2013:247:292-5.

33. Zappia E, Casazza S, Pedemonte E, Benvenuto F, Bonanni I, Gerdoni E, et al. Mesenchymal stem cells ameliorate experimental autoimmune encephalomyelitis inducing T-cell anergy. Blood. 2005;106(5):1755-61.

34. Gerdoni E, Gallo B, Casazza S, Musio S, Bonanni I, Pedemonte E, et al. Mesenchymal stem cells effectively modulate pathogenic immune response in experimental autoimmune encephalomyelitis. Ann Neurol. 2007:61(3):219-27.

35. Le Blanc K, Samuelsson H, Gustafsson B, Remberger M, Sundberg B, Arvidson J, et al. Transplantation of mesenchymal stem cells to enhance engraftment of hematopoietic stem cells. Leukemia. 2007;21(8):1733-8.

36. Lazarus HM, Koc ON, Devine SM, Curtin P, Maziarz RT, Holland HK, et al. Cotransplantation of HLA-identical sibling culture-expanded mesenchymal stem cells and hematopoietic stem cells in hematologic malignancy patients. Biol Blood Marrow Transplant. 2005;11(5):389-98. 
37. Le Blanc K, Frassoni F, Ball L, Locatelli F, Roelofs H, Levis I, et al. Mesenchymal stem cells for treatment of steroid-resistant, severe, acute graft-versus-host disease: a phase II study. Lancet. 2008;371:1579-86.

38. Krampera M, Glennie S, Dyson J, Scott D, Laylor R, Simpson E, et al. Bone marrow mesenchymal stem cell inhibit the response of naïve and memory antigen-specific T cells to their cognate peptide. Blood. 2003;101(9):3722-9.

39. Augello A, Tasso R, Negrini SM, Amateis A, Indiveri F, Cancedda R, et al. Bone marrow mesenchymal progenitor cells inhibit lymphocyte proliferation by activation of the programmed death 1 pathway. Eur J Immunol. 2005:35(5):1482-90.

40. Krampera $M$, Cosmi L, Angeli R, Pasini A, Liotta F, Andreini A, et al. Role for interferon-gamma in the immunomodulatory activity of human bone marrow mesenchymal stem cells. Stem Cells. 2006;24(2):386-98.

41. Ren G, Zhang L, Zhao X, Xu G, Zhang Y, Roberts Al, et al. Mesenchymal stem cells mediated immunosuppression occurs via concerted action of chemokines and nitric oxide. Cell Stem Cells. 2008;2(2):141-50.

42. Vergani L, Lanza C, Rivaro P, Abelmoschi LM, Genti S, Veneselli E, et al. Metals, metallothioneins and oxidative stress in blood of autistic children. Res Autism Spectr Disord. 2011;5(1):286-93.

43. Grasselli E, Voci A, Canesi L, De Matteis R, Goglia F, Cioffi F, et al. Direct effects of iodothyronines on excess fat storage in rat hepatocytes. J Hepatol. 2011;54(6):1230-6.

44. Pfaffl MW. A new mathematical model for relative quantification in real-time RT-PCR. Nucleic Acids Res. 2001;29(9), e45.

45. Vignali DA. Multiplexed particle-based flow cytometric assays. J Immunol Methods. 2000;243(1-2):243-55.

46. Corcione A, Arduino N, Ferretti PA, Spinelli M, Ottonello L, et al. Chemokine receptor expression and function in childhood acute lymphoblastic leukemia of B-lineage. Leuk Res. 2006;30:365-72.

47. Constantin G. Chemokine signaling and integrin activation in lymphocyte migration into the inflamed brain. J Neuroimmunol. 2008;198(1-2):20-6.

48. Freedman MS, Uccelli A. Neurorepair with mesenchymal stem cells: hope or hype? Lancet Neurol. 2012;11(2):123-5.

49. Glennie S, Soeiro I, Dyson PJ, Lam EW, Dazzi F. Mesenchymal stem cells induce division arrest anergy of activated T cells. Blood. 2005;105(7):2821-7.

50. Harrer A, Wipfler P, Einhaeupl MH, Pilz G, Oppermann K, Hitzl W, et al. Natalizumab therapy decreases surface expression of both VLA-heterodimer subunits on peripheral blood mononuclear cells. J Neuroimmunol. 2011;234(1-2):148-54.

51. van Kooyk $Y$, van de Wiel-van Kemenade $P$, Weder $P$, Kuijpers TW, Figdor CG. Enhancement of LFA-1-mediated cell adhesion by triggering through CD2 or CD3 on T lymphocytes. Nature. 1989;342(6251):811-3.

52. Vazirinejad R, Ahmadi Z, Kazemi Arababadi M, Hassanshahi G, Kennedy D. The biological functions, structure and sources of CXCL10 and its outstanding part of the pathophisiology of multiple sclerosis. Neuroimmunomodulation. 2014;21(6):322-30.

53. Ramasamy R, Tong CK, Seow HF, Vidyadaran S, Dazzi F. The immunosuppressive effects of human bone marrow-derived mesenchymal stem cells target $T$ cell proliferation but not its effector function. Cell Immunol. 2008;251(2):131-6.

54. Arimilli S, Ferlin W, Solvason NA, Deshpande S, Howard M, Mocci S. Chemokines in autoimmune diseases. Immunol Rev. 2000;177:43-51.

55. Liu L, Huang D, Matsui M, He T, Hu T, Demartino J, et al. Severe disease, unaltered leukocyte migration, and reduced IFN-gamma production in CXCR3-/- mice with experimental autoimmune encephalomyelitis. J Immunol. 2006;176(7):4399-409.

56. Müller M, Carter SL, Hofer MJ, Manders P, Getts DR, Getts MT, et al. CXCR3 signaling reduces the severity of experimental autoimmune encephalomyelitis by controlling the parenchymal distribution of effector and regulatory T cells in the central nervous system. J Immunol. 2007;179(5):2774-86.

57. Kawai K, Kobayashi Y, Shiratori M, Sobue G, Tamatani T, Miyasaka M, et al. Intrathecal administration of antibodies against LFA-1 and against ICAM-1 suppresses experimental allergic encephalomyelitis in rats. Cell Immunol. 1996;171(2):262-8.

58. Willenborg DO, Simmons RD, Tamatani T, Miyasaka M. ICAM-1-dependent pathway is not critically involved in the inflammatory process of autoimmune encephalomyelitis or in cytokine-induced inflammation of the central nervous system. J Neuroimmunol. 1993;45(1-2):147-54.
59. Kobayashi Y, Kawai K, Honda H, Tomida S, Niimi N, Tamatani T, et al. Antibodies against leukocyte function-associated antigen-1 and against intercellular adhesion molecule-1 together suppress the progression of experimental allergic encephalomyelitis. Cell Immunol. 1995;164(2):295-305.

60. Samoilova EB, Horton JL, Chen Y. Experimental autoimmune encephalomyelitis in intercellular adhesion molecule-1-deficient mice. Cell Immunol. 1998;190(1):83-9.

61. Bullard DC, Hu X, Schoeb TR, Collins RG, Beaudet AL, Barnum SR. Intercellular adhesion molecule-1 expression is required on multiple cell types for the development of experimental autoimmune encephalomyelitis. J Immunol. 2007;178(2):851-7.

62. Hu X, Barnum SR, Wohler JE, Schoeb TR, Bullard DC. Differential ICAM-1 isoform expression regulates the development and progression of experimental autoimmune encephalomyelitis. Mol Immunol. 2010;47(9):1692-700.

63. Swart GW. Activated leukocyte cell adhesion molecules (ALCAM/CD166): developmental and mechanistic aspects of cell-clustering and cell migration. Eur J Cell Biol. 2002;81(6):313-21.

\section{Submit your next manuscript to BioMed Central and take full advantage of:}

- Convenient online submission

- Thorough peer review

- No space constraints or color figure charges

- Immediate publication on acceptance

- Inclusion in PubMed, CAS, Scopus and Google Scholar

- Research which is freely available for redistribution 